This item was submitted to Loughborough's Research Repository by the author.

Items in Figshare are protected by copyright, with all rights reserved, unless otherwise indicated.

\title{
Communication and interaction with semiautonomous ground vehicles by force control steering
}

PLEASE CITE THE PUBLISHED VERSION

https://doi.org/10.1109/tcyb.2020.3020217

\section{PUBLISHER}

Institute of Electrical and Electronics Engineers (IEEE)

\section{VERSION}

AM (Accepted Manuscript)

\section{PUBLISHER STATEMENT}

(c) 2020 IEEE. Personal use of this material is permitted. Permission from IEEE must be obtained for all other uses, in any current or future media, including reprinting/republishing this material for advertising or promotional purposes, creating new collective works, for resale or redistribution to servers or lists, or reuse of any copyrighted component of this work in other works.

\section{LICENCE}

\section{All Rights Reserved}

\section{REPOSITORY RECORD}

Martinez-Garcia, Miguel, Roy Kalawsky, Timothy Gordon, Tim Smith, Qinggang Meng, and Frank Flemisch. 2020. "Communication and Interaction with Semiautonomous Ground Vehicles by Force Control Steering". Loughborough University. https://hdl.handle.net/2134/13026362.v1. 


\title{
Communication and Interaction With Semiautonomous Ground Vehicles by Force Control Steering
}

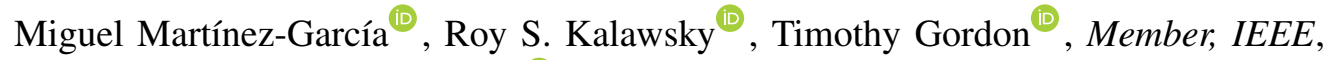 \\ Tim Smith, Qinggang Meng ${ }^{\mathbb{1}}$, Senior Member, IEEE, and Frank Flemisch
}

\begin{abstract}
While full automation of road vehicles remains a future goal, shared-control and semiautonomous drivinginvolving transitions of control between the human and the machine-are more feasible objectives in the near term. These alternative driving modes will benefit from new research toward novel steering control devices, more suitably where machine intelligence only partially controls the vehicle. In this article, it is proposed that when the human shares the control of a vehicle with an autonomous or semiautonomous system, a force control, or nondisplacement steering wheel (i.e., a steering wheel which does not rotate but detects the applied torque by the human driver) can be advantageous under certain schemes: tight rein or loose rein modes according to the $\boldsymbol{H}$-metaphor. We support this proposition with the first experiments to the best of our knowledge, in which human participants drove in a simulated road scene with a force control steering wheel (FCSW). The experiments exhibited that humans can adapt promptly to force control steering and are able to control the vehicle smoothly. Different transfer functions are tested, which translate the applied torque at the FCSW to the steering angle at the wheels of the vehicle; it is shown that fractional order transfer functions increment steering stability and control accuracy when using a force control device. The transition of control experiments is also performed with both: a conventional and an FCSW. This prototypical steering system can be realized via steer-by-wire controls, which are already incorporated in commercially available vehicles.
\end{abstract}

Index Terms-Cybernetics, ground vehicle automation, haptics, human-machine integration, steer-by-wire (SBW), steering control.

Manuscript received January 10, 2020; revised May 24, 2020; accepted August 21, 2020. This article was recommended by Associate Editor Z. Ju. (Corresponding author: Miguel Martínez-García.)

Miguel Martínez-García is with the Department of Aeronautical and Automotive Engineering, Loughborough University, Loughborough LE11 3TU, U.K. (e-mail: m.martinez-garcia@lboro.ac.uk).

Roy S. Kalawsky is with the Advanced Virtual Reality Research Centre, Wolfson School of Mechanical, Electrical and Manufacturing Engineering, Loughborough University, Loughborough LE11 3TU, U.K. (e-mail: r.s.kalawsky@lboro.ac.uk).

Timothy Gordon and Tim Smith are with the School of Engineering, University of Lincoln, Lincoln LN6 7TS, U.K. (e-mail: tgordon@lincoln.ac.uk; tismith@lincoln.ac.uk).

Qinggang Meng is with the Department of Computer Science, Loughborough University, Loughborough LE11 3TU, U.K. (e-mail: q.meng@lboro.ac.uk).

Frank Flemisch is with the Department of Ergonomics and Human Machine Systems Research Area, Fraunhofer Institute for Communication, Information Processing and Ergonomics FKIE, 53343 Wachtberg, Germany (e-mail: f.flemisch@iaw.rwth-aachen.de).

Color versions of one or more of the figures in this article are available online at http://ieeexplore.ieee.org.

Digital Object Identifier 10.1109/TCYB.2020.3020217

\section{INTRODUCTION}

\section{A. Background}

D URING recent years, news broadcasts of science and technology trends have placed a special interest in the possibility of driverless cars pervading public roads. Despite the eager news coverage, fully autonomous vehicles may not materialize in the short term-or not materialize at all [1]. There are a number of technical challenges that presently do not have a clear solution. Conceivably, the most hindering obstacles are amidst the domain of artificial intelligence (AI); the way in which artificial neural networks generalize is not yet fully understood [2], and they can be easily fooled by perturbations in the input data [3].

Although conventional cars will not attain full automation anytime soon, early generations of associated developments will potentially result. An obvious one is the occurrence of autonomous transportation systems within restricted environments. Driverless cars in simplified environments may be implemented. This can be accomplished by adapting the infrastructure. For instance, eLanes could be established in which only vehicles in the autonomous control mode are allowed to circulate [4]. The eLane design would conform to the operational design domain (ODD) of the vehicle. Entering or departing eLanes will entail transitions of control between the human and the machine. Another interesting prospect is that of shared-control systems, where the control of the machine is shared between the human and the intelligent system simultaneously [1].

The idea of shared control has been present in the literature for quite some time, yet presently, it is gaining momentum. Within the classical literature, the paper by Birmingham and Taylor on human-machine systems [5] is an illustrative case; it is affirmed that, because of the high adaptability that humans exhibit, they should never be removed from the control loop. Instead, this article suggests that the system design needs to be aimed toward unburdening the human operator. Thus, it promotes the concept of using transfer functions between the control device and the machine, with the intent of facilitating the operator task. A similar notion is highlighted in a later publication by Licklider, through an alternative strategy. In [6], it was proposed that the human operator can be temporarily removed from the control loop, 
but needs to be ready to "...handle the very-low-probability situations...," for which the autonomous system was not designed for. Another shared-control approach is the use of haptic-guidance [7], [8], in which human performance is enhanced through force feedback.

Since these ideas have been present in the literature for decades, why is the research related to these concepts relevant these days? Several recent technological advances help to answer this question. One of them is the so-called by-wire controls, which substitute the mechanical connection from a control device to the vehicle actuators with an electronic subsystem. Control by-wire has existed in the aerospace industry since the $1980 \mathrm{~s}$, but it is only recently that it was incorporated into the automotive industry; in 2014, the first commercially available car with steer-by-wire (SBW) control was releasedthe Nissan Infinity Q50. Thus, designing transfer functions between the human and the vehicle-to unburden the human operator-have attained general practicability. SBW systems allow for the integral replacement of conventional steering systems with new synthetic mappings.

A relevant question, tackled in this article, is the role of the steering wheel in semiautonomous vehicles. The lateral dynamics of cars has been commonly handled with a steering wheel since its inception. Considering that the control of ground vehicles may be shared between the human and the machine in the future, a conventional steering wheel may not be the best interface device. An elucidating analogy is that of horse-drawn vehicles, such as horsecars or stagecoaches, which essentially are shared-control systems where the control is shared between the human and the horse [9], [10]; although the human is in charge of the decisions at the tactical and strategic levels [11], the horse has a certain autonomy at the operational level.

In this article, the novel concept of a nondisplacement steering wheel, or force control steering wheel (FCSW), was examined, as a substitute for the traditional steering system for autonomous and semiautonomous vehicles. An FCSW is a steering wheel that does not rotate but detects the applied torque by the human driver. Although new for highway driving, the concept of force control has been applied in the past in a variety of different contexts.

\section{B. Related Work}

The force control devices that have been tested and/or employed until now, are control sticks (or joysticks) and pointing sticks (or trackpoints). One of the earliest appearances of a force control stick in the literature is found in [12], where force control was applied through a lever in a tracking task and compared with that of a moving lever. The plants controlled by the lever represented proportional, rate, and acceleration control; interestingly, force control was shown to be more efficient. In the same publication, it is also reported that the participants learned to manipulate the force control device more rapidly. With a similar experimental apparatus, and some years later, McRuer communicated comparable results [13]. For the experiments reported in both publications, the test participants were skilled at tracking tasks-naval officers and pilots.
In [14], a rate force control joystick - thumb controlledwas compared with a computer mouse and with the arrow keys of a computer keyboard. In this case, it was concluded that the quickest performance and the lowest error are produced with the computer mouse. Nevertheless, in this case, the task at hand did not consist of a tracking exercise; it was a pointing task in which test participants had to select text in a display by displacing a cursor.

In a later paper [15], the comparison between a force control device and a computer mouse - also for pointing tasks - was further studied; it was shown that for pointing tasks, a computer mouse is more efficient than a pointing stick, but for the case when pointing is combined with typing, the pointing stick is slightly superior. In the same paper, transfer functions between the forces applied to the device and the pointer speed of linear, parabolic, and sigmoid shapes were tested. It was concluded that the sigmoid transfer function yielded the best results. In this case, the force controller was a pointing stick very similar to what today is still incorporated into the ThinkPad laptops, for that research is related to the development of those particular devices by IBM.

ThinkPad trackpoints are perhaps the most well-known application of a force control device to date. They are handled through a small hard rubber pad, thus they constitute minimum-displacement sticks. These trackpoints also incorporate a lead-lag compensator producing a negative inertia effect to increase pointing performance [16], ${ }^{1}$ but such an approach is not suitable for when overshooting can produce a fatal accident.

Besides IBM's pointing sticks, there have been other examples of technology products containing some sort of force controller, such as the side-sticks in the F-16 fighter aircraft [18], and the $C$-stick included in some Nintendo videogame systems, which is used to pan the camera and adjust the field of view. Also, an FCSW was previously utilized in [19] to assess the effects of steering feel, but not as a steering control device for vehicle driving.

In all the discussed cases, the design of the mapping between the applied gain at the force control device and the resultant output was essential. This mapping is highly dependent on the particular control device used, and on the characteristics of the control task.

\section{Contribution}

In this article, various hypotheses are studied with respect to driving a ground vehicle with an FCSW. First, it is assessed whether humans are able to drive a simulated vehicle in a typical road scenario by torque or force control steering (FCS). Tests were conducted at different speeds. Several transfer functions, mapping the applied torque to the steering angle at the wheels of the vehicle, were investigated: constant gain, proportional-integral (PI) filters, and fractional order filters.

Another concerning condition, in autonomous driving systems research, is that of control transitions. The driver's reaction time to a potential hazard is typically larger when

\footnotetext{
${ }^{1}$ The concept of negative inertia had been already explored much before by Tustin [17].
} 
control transitions are involved [20]. Thus, one of the questions investigated in this article is the possibility of reducing the reaction time of the driver, when the human regains control from an autonomous system, with an FCSW. To the best of our knowledge, this is the first study in which such FCS experiments are reported. In summary, the contributions of this work are as follows.

1) Experiments are conducted to showcase the capability of humans in learning and performing FCS.

2) Suitable transfer functions are identified, to transduce the applied torque by the human driver into a steering wheel angle. These are based on fractional order filters.

3) Additional experiments are conducted to assess the efficacy of FCS when the human has to regain control from an autonomous system.

4) Design guidelines are provided to employ FCS in various shared-control schemes.

The article is organized as follows. In Section II, the methodology, experimental setup, participants, and experiments are described. These experiments include an adaptation phase and a driving phase. The results from the experiments are examined in Section III. In Section IV, the experiments are discussed conceptually, and a summary of some of the potential applications of FCS, not explicitly studied experimentally in the article, are provided. Final conclusions are drawn in Section V.

\section{Methods}

Naturalistic driving data are inherently noisy and difficult to analyze [21]. On the other hand, data collected from participants in laboratory tasks, although maybe less representative of realistic driving, are more interpretable. For this research, an experimental setup consisting of a simple driving simulator-presenting the forward view scene of a roadway from a ground vehicle- and an FCSW were implemented. In addition, a Logitech G27 steering wheel was utilized with the same software, as a baseline for comparing performance (Fig. 1). The leading objective of the experiments was to assess and to compare human performance between both control devices under various conditions: simple tracking tasks, ground vehicle driving at different speeds, and regain of control from an autonomous system. For the case of FCS, several transfer functions- translating applied torque to steering wheel angle-are investigated, including fractional order transfer functions.

\section{A. Experimental Setup}

1) Roadway-Vehicle Simulation: A computer simulation was designed for the purpose of performing the experiments. The computer graphics were generated in 3-D with the open graphics library (OpenGL) API [22]. The display was refreshed at a variable frame rate (of at least $40 \mathrm{~Hz}$ ); this minimum rate suffices to ensure ideal control performance [23]. The graphical simulation consisted of a forward road scene [Fig. 4(a)] with varying road geometry for the different experiments. The simulation runs in real time at $1000 \mathrm{~Hz}$. At each time step, the states of the vehicle simulation were updated

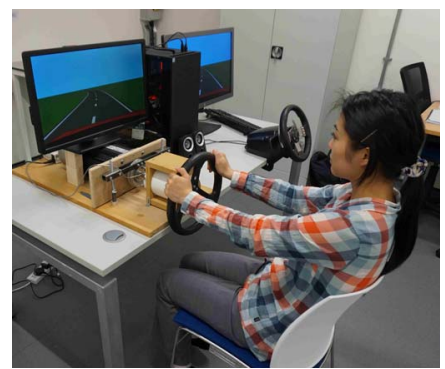

(a)

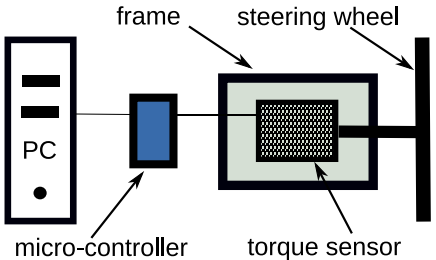

(b)
Fig. 1. (a) Experimental setup as presented to the participants. In front of the left display, the FCSW is mounted on a frame. On the right display, the Logitech G27 is mounted. (b) Schematic of the FCSW; the steering wheel was locked and attached to a torque sensor in a frame. A microcontroller transferred the sensor readings to a desktop PC. Both control devices interact with the same simulation software on a desktop PC.

TABLE I

Vehicle Model Parameters as Set for the Driving SIMULATOR EXPERIMENTS

\begin{tabular}{|l||c|}
\hline Mass of the vehicle $M$ & $1500 \mathrm{~kg}$ \\
\hline Distance from vehicle CG to front axle $l_{f}$ & $1.1 \mathrm{~m}$ \\
\hline Distance from vehicle CG to rear axle $l_{r}$ & $1.6 \mathrm{~m}$ \\
\hline Front cornering stiffness (both wheels) $C_{\alpha f}$ & $55000 \mathrm{~N} / \mathrm{rad}$ \\
\hline Rear cornering stiffness (both wheels) $C_{\alpha r}$ & $60000 \mathrm{~N} / \mathrm{rad}$ \\
\hline Yaw moment of inertia $I$ & $2500 \mathrm{~kg} \cdot \mathrm{m}^{2}$ \\
\hline Steering ratio $r_{s}$ & $16: 1$ \\
\hline
\end{tabular}

through a Runge-Kutta method of order $\mathcal{O}\left(h^{4}\right)$ [24]. The vehicle states were the body slip angle $\beta(t)$ and the yaw rate $\eta(t)$, and were simulated with the linear single-track vehicle model found in [25] - (1) and (2) with the parameters in Table I

$$
\begin{aligned}
{\left[\begin{array}{c}
\dot{\beta} \\
\dot{\eta}
\end{array}\right]=} & -\left[\begin{array}{cc}
L_{0} / M U & 1+L_{1} / M U^{2} \\
L_{1} / I & L_{2} / I U
\end{array}\right]\left[\begin{array}{l}
\beta \\
\eta
\end{array}\right] \\
& +\left[\begin{array}{c}
C_{\alpha f} / M U \\
l_{f} C_{\alpha f} / I
\end{array}\right] \delta
\end{aligned}
$$

with

$$
\left\{\begin{array}{l}
L_{0}=C_{\alpha f}+C_{\alpha r} \\
L_{1}=l_{f} C_{\alpha f}+l_{r} C_{\alpha r} \\
L_{2}=l_{f}^{2} C_{\alpha f}+l_{r}^{2} C_{\alpha r}
\end{array}\right.
$$

where $C_{\alpha f}$ and $C_{\alpha r}$ are the axle cornering stiffness for the front and rear axles, respectively, $M$ is the mass of the vehicle, $U$ is the speed-which was a constant value for each experiment, $l_{f}$ and $l_{r}$ are the distances from the center of gravity (CG) of the vehicle to the front and rear axles, respectively, $I$ is the yaw moment of inertia, and $\delta$ is the steering angle at the front wheels.

The recorded variables were the simulation time, vehicle position, vehicle heading, yaw rate, lateral offset, body slip angle, steering angle (or torque, depending on the employed control device-Section II-A2). While ground vehicles typically display nonlinear dynamics, these do not largely differ from the linear regime. For the analysis of steering control, linear vehicle models are sufficient. 
TABLE II

SPECIFICATIONS OF THE TORQUe SENSOR AND THE FCSW

\begin{tabular}{|l||c|}
\hline Torque Sensor Model & Omega TQ301-45N \\
\hline Sensor range & $0-45 \mathrm{~N} \cdot \mathrm{m}$ \\
\hline Sensor reading range - limited & $0-15 \mathrm{~N} \cdot \mathrm{m}$ \\
\hline Accuracy & $\pm 0.2 \% \mathrm{FSO}$ \\
\hline Steering wheel diameter & $0.32 \mathrm{~m}$ \\
\hline
\end{tabular}

2) Hardware: Two displays and two control devices-one display for each control device-were connected to the computer that runs the simulation (Fig. 1). Speakers were used to signal the driver of varying conditions during the experiments. The two control devices were a Logitech G27 steering wheel and an FCSW. The motor of the Logitech G27 was controlled from the simulation application to produce a selfaligning torque effect, which was proportional to the lateral force on the front tires [25].

The FCSW was built for this particular experiment and consisted of a torque sensor (Table II) attached to a support frame [Fig. 1(b)]. The signal from the torque sensor was read and conditioned by a single-board microcontroller. The simulation application requested the state of the sensor to the microcontroller at each simulation step. When requested, the microcontroller broadcasted the torque sensor back to the application. The communication between the devices was set at 921600 baud to achieve negligible latency.

The steering wheel was configured to rotate $900^{\circ}$ lock to lock, corresponding to $|\delta| \leq 28.13^{\circ}$ with the chosen steering ratio (Table I). When driving by torque with the FCSW, these maximum values for $\delta$ were achieved with applied torque of $\pm 15 \mathrm{~N} \cdot \mathrm{m}( \pm 4.78 \mathrm{~kg})$ - which was the magnitude limit when reading from the torque sensor (Table II).

\section{B. Fractional Order Filters}

Different types of transfer functions-mapping the input to the control device by the human operator to the vehicle model-were considered. Among the candidates were fractional order transfer functions. ${ }^{2}$ In the fractional calculus approach, the traditional notions of integral and derivative are extended to define the noninteger powers of the integral and differential operators [26].

For the case of fractional order integration of order $v>0$ of a function $f$-denoted as $D^{-v} f(t)$ - the Laplace transform is given by the relation

$$
\mathcal{L}\left\{D^{-v} f(t)\right\}=\frac{F(s)}{s^{v}}
$$

where $\mathcal{L}\{f(t)\}=F(s)$. The frequency response of $1 / s^{v}$ with $v \in \mathbb{R}$ is shown in Fig. 2. Thus for $v=1$, (3) corresponds to the classical first-order integration: $D^{-1} f(t)=\int_{0}^{t} f(t)$.

Fractional order transfer functions have been favorably used for modeling biological systems [27]. In [28], they were employed for modeling human operators controlling vehicles in a cybernetical control loop [29]. Herein, the advantage of

\footnotetext{
${ }^{2}$ For this research, the Riemann-Liouville fractional integral was employed [26].
}

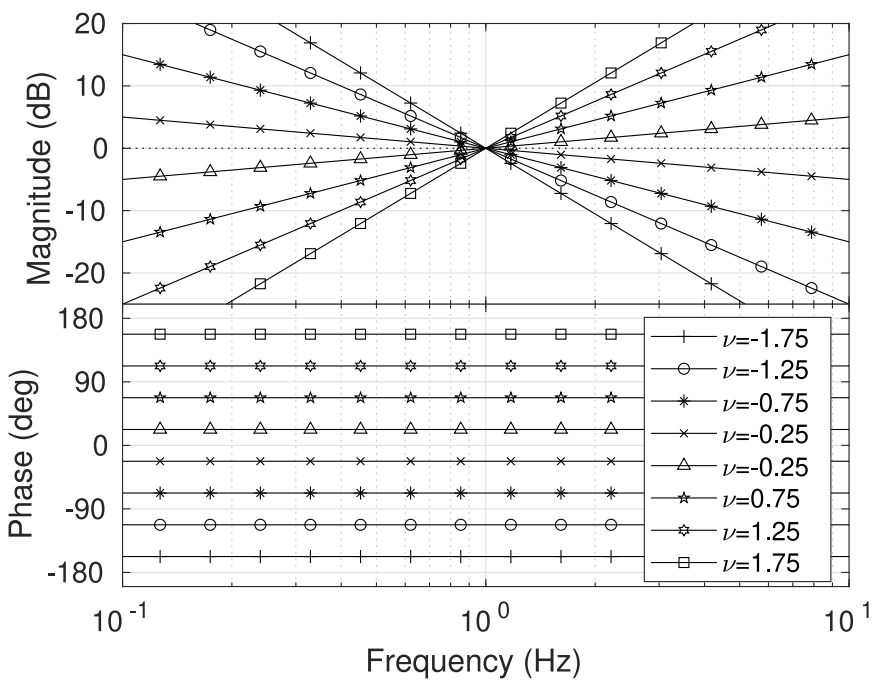

Fig. 2. Frequency response of $1 / s^{v}$ by varying $v$. The slope of the magnitude response is $v \cdot 20 \mathrm{~dB} /$ decade while the phase response is $\nu \cdot \pi / 2$ rad for $v \in \mathbb{R}$.

TABLE III

Gender and Age of the Ten Participants in the Experiments. IN THE Table, It Is Also Detailed Whether the Participants Hold a DRIVING LICENSE OR NOT, THEIR DRIVING EXPERIENCE (THAT IS, THEY Drive At Least Once a Week), and if They Play Videogames FREQUENTLY (THAT IS, AT LEAST ONCE A MONTH)

\begin{tabular}{|r||c|c|c|c|c|}
\hline PAT. & Age & Gender & D. Lic. & D. Exp & V. Exp \\
\hline \hline P1 & 32 & M & $\checkmark$ & $\checkmark$ & $\times$ \\
\hline P2 & 33 & M & $\checkmark$ & $\checkmark$ & $\checkmark$ \\
\hline P3 & 34 & F & $\times$ & $\times$ & $\times$ \\
\hline P4 & 41 & M & $\checkmark$ & $\checkmark$ & $\times$ \\
\hline P5 & 25 & F & $\checkmark$ & $\checkmark$ & $\times$ \\
\hline P6 & 28 & M & $\checkmark$ & $\checkmark$ & $\times$ \\
\hline P7 & 22 & M & $\checkmark$ & $\checkmark$ & $\checkmark$ \\
\hline P8 & 20 & M & $\checkmark$ & $\checkmark$ & $\checkmark$ \\
\hline P9 & 30 & M & $\checkmark$ & $\checkmark$ & $\times$ \\
\hline P10 & 28 & F & $\checkmark$ & $\checkmark$ & $\times$ \\
\hline
\end{tabular}

this method relies on that it allows for the introduction of integration in gradual noninteger increments-hence it is convenient for laboratory tasks. Fractional operators provide a simple tuning procedure for adjusting the level of haptic feel, as they are described by only one parameter, which can be interpreted as the amount of memory introduced in the system. In [30], the technical background for this approach is discussed in detail.

\section{Participants}

Ten participants of varying age, gender, and level of driving experience completed the experiments (Table III). All the participants joined voluntarily and, prior to the experiments, signed a consent form. ${ }^{3}$

The experiments were executed over two different days, to monitor human adaptation to FCS. Each day comprised approximately $30 \mathrm{~min}$ of driving with some periods of rest. In

\footnotetext{
${ }^{3}$ These tests were approved by the College of Science Research Ethics Committee of the University of Lincoln (U.K.) with UID COSREC491.
} 


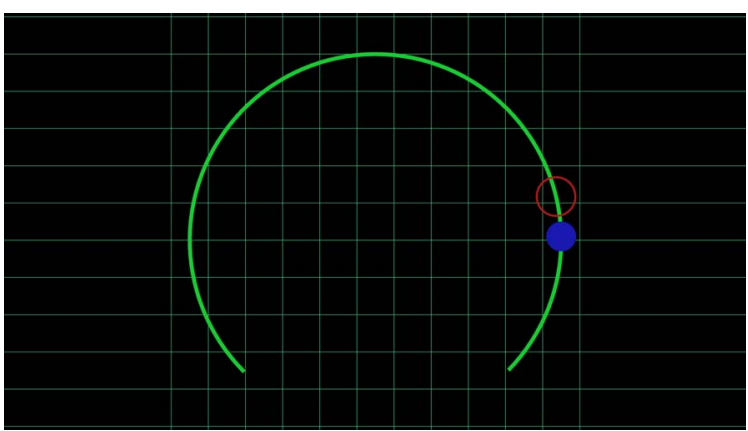

Fig. 3. Visual display as in the tracking experiments \#1 and \#2 during day 1. The participants had to apply torque to the FCSW to position the cursor (blue dot) inside the target (red circle)—which varied its position at each time interval. The cursor and target moved only over the circular arc (green dial), with a rest state at its topmost position.

all the driving experiments, the visual projection represented a driver view from the left seat of the car-with an offset of $0.45 \mathrm{~m}$. The experiments in day 1 included a simple tracking task so that the participants could adapt to the FCSW.

\section{Training Experiments Phase-Day 1}

1) Tracking Experiment \#1: In the first tracking experiment, a red target circle was presented in the display, which varied position along an arc between two alternate locationscorresponding to a torque of $\pm 10.5 \mathrm{~N} \cdot \mathrm{m}$ (Fig. 3). The target changed its position every $20 \mathrm{~s}$, and the total duration of the experiment was $180 \mathrm{~s}$. A blue-colored dot represented the applied torque to the FCSW in the display. The participants were requested to apply force on the FCSW in order to place the cursor (blue dot) in the center of the target (red circle).

2) Tracking Experiment \#2: The second FCS tracking experiment was very similar to the first and had the same duration (180 s). The only differences were that the target changed position more frequently-every $6 \mathrm{~s}$ - and at randomized locations over the dial.

During the training phase (day 1) other experiments were included which involved driving with the Logitech G27 and with the FCSW in tracks of different geometry. These experiments were also performed for adaptation purposes, thus are not explicitly described here. Nevertheless, some of these experiments are compared with those of day 2 in Section III.

\section{E. Performance Measurement Experiments-Day 2}

The experiments in day 2 comprised driving at different speeds with the FCSW and a regain of the control experiment, which was carried out with both control devices (steering wheel and FCSW).

The participants drove with the FCSW in a simulated road scene (Section II-A1) through a pseudorandomly generated road at 30,50, and $70 \mathrm{~km} / \mathrm{h}$. Each experiment was performed at a constant speed. While this is not a fully realistic driving scenario, the recorded signals can be considered stationary and comparisons among subjects are feasible. The road geometry was produced by a Perlin noise generator [31] [Fig. 4(a)]. The emphasis of these experiments was placed on investigating potential transfer functions between the torque sensor and

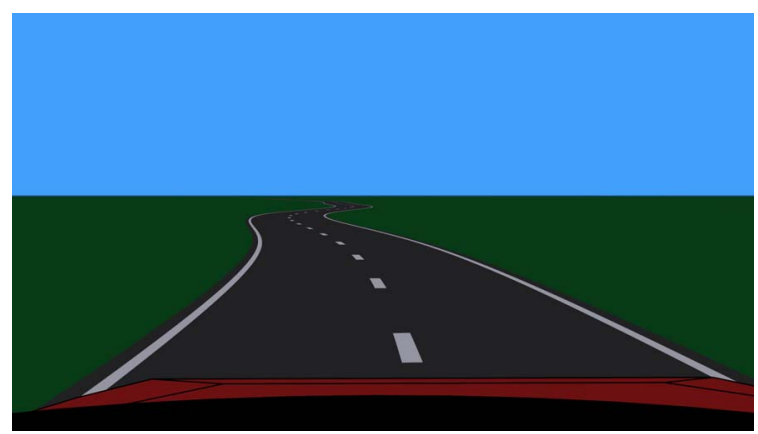

(a)

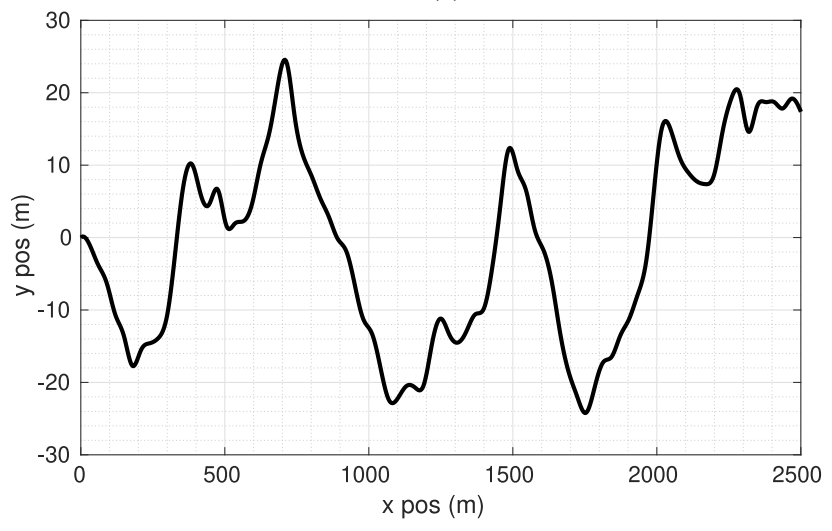

(b)

Fig. 4. (a) Forward view of the simulated road in the driving experiments (b) Sample of the road geometry for a pseudorandomly generated road-the Perlin noise.

TABLE IV

TRANSFER FunCTIONS OF THE FILTERS EMPLOYED DURING THE FCS EXPeriments That Translate Torque to SteEring WheEl ANGLE

\begin{tabular}{|l||c|c|c|}
\hline & Gain & Fractional & PI \\
\hline speed & $K_{p}$ & $\frac{1}{s^{\nu}}$ & $K+(1-K) \frac{1}{s}$ \\
\hline \hline $30 \mathrm{~km} / \mathrm{h}$ & \multirow{3}{*}{0.75} & $\nu=0.05$ & $K=0.5$ \\
\cline { 1 - 1 } $50 \mathrm{~km} / \mathrm{h}$ & $\nu=0.25$ & $K=0.6$ \\
\cline { 1 - 1 } $70 \mathrm{~km} / \mathrm{h}$ & & $\nu=0.35$ & $K=0.7$ \\
\hline
\end{tabular}

the vehicle model. These are summarized in Table IV and discussed in more detail in the following.

As a baseline, a transfer function consisting only of a constant gain was first considered. The value of this gain, and the values of the parameters of the other transfer functions, were tuned empirically before the experiments by informal testing by the researchers. This was done so that the number of experimental variants presented to the participants was kept at an acceptable level; the parameters were identical for all the participants (Table IV).

A fractional order integrator was also considered as the candidate transfer function-(3). As the experiments involved driving within the simulation at distinct speeds, the index of fractional integration was tuned empirically for each speed value (Table IV). In prior informal tests, it was observed that a higher order of fractional integration was better for higher speeds. In addition, to contrast the effects of the memory added 
TABLE V

Filter Used at Each of the Phases of THE DRIVING EXPERIMENTS With the FCSW. Their SPECIFICS ARE Defined IN TABLe IV

\begin{tabular}{|l||l|c|}
\hline Phase \# & Transfer Function & Duration \\
\hline \hline 1 & Gain: $K_{p}$ & $60 \mathrm{~s}$ \\
\hline 2 & Fractional: $1 / s^{\nu}$ & $60 \mathrm{~s}$ \\
\hline 3 & PI: $K+(1-K) \frac{1}{s}$ & $60 \mathrm{~s}$ \\
\hline 4 & Fractional: $1 / s^{\nu}$ & $60 \mathrm{~s}$ \\
\hline 5 & PI: $K+(1-K) \frac{1}{s}$ & $60 \mathrm{~s}$ \\
\hline
\end{tabular}

by the fractional operator with a standard approach, a PI filter was also examined. The PI filter was constrained to a single parameter, to match the interpretation with the fractional filter. As the PI filter involves a pure integral with a very extreme memory effect-as compared to low-level fractional integration-it was observed that lowering the effect of the integrator was seemingly better at higher speeds (Table IV).

1) FCS at 30km/h: This experiment consisted of driving during 5 min with an FCSW on a simulated roadway-which was the same for all the participants (Fig. 4). To avoid the introduction of bias by the particular road geometry, half of the participants drove the road in the opposite direction as compared to the other half. The simulation represented a vehicle with a constant speed of $30 \mathrm{~km} / \mathrm{h}$ and the driver sitting at the left seat of the car. Every $60 \mathrm{~s}$, an acoustic signal and an intermittent change in color in the background sky indicated to the driver that a change in the dynamics of the vehicle had occurred; the test participants had been informed about this procedure before the experiment was conducted. Also, the participants had performed a similar experiment in day 1 . The change in the dynamics reflected a different transfer function between the torque sensor and the vehicle model. The order in which the transfer functions were presented is specified in Table V. Thus, the experiment consisted of five phases.

2) FCS at $50 \mathrm{~km} / \mathrm{h}$ : This experiment is analogous to the one above (Section II-E1), with the exception of the vehicle speed, which in this case was $50 \mathrm{~km} / \mathrm{h}$. An identical experiment to this one had been performed in day 1 , as part of the adaptation process to the FCSW. During the first day of the experiments, the participants drove the simulation with the steering wheel, on the same road, and at the same speed.

3) FCS at $70 \mathrm{~km} / \mathrm{h}$ : The same experiment as above (Section II-E1) was also performed at $70 \mathrm{~km} / \mathrm{h}$.

4) Regain of Control Experiments: These experiments were designed to test if the reaction time of the human driver when regaining control from an autonomous system, can be reduced with an FCSW. For this, two essentially identical experiments were executed by every participant, in which the only difference was the employed control device (steering wheel or FCSW).

In the regain of control experiments, the participants were asked to look at the OpenGL simulation while the vehicle drove autonomously through a curved road geometry ${ }^{4}$ (Fig. 5), while keeping the hands off the steering wheel (or FCSW).

\footnotetext{
${ }^{4}$ This was done through the implementation of the Salvucci and Gray model [32].
}

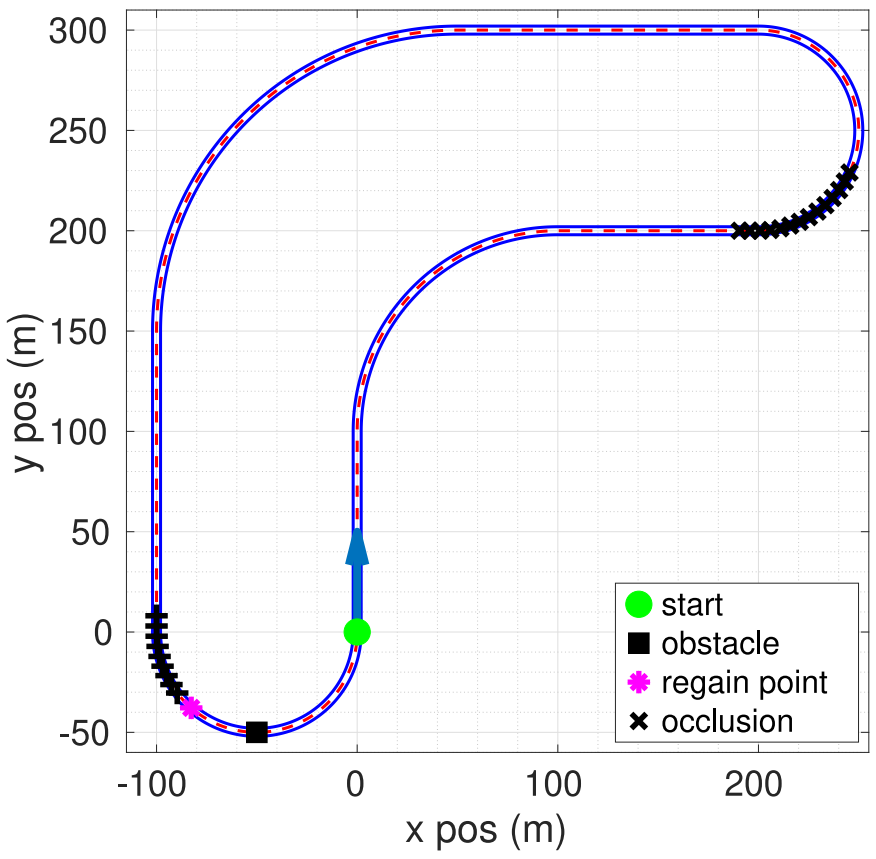

Fig. 5. Road geometry in the regain of control experiments, displaying the occluded regions, the obstacle position, and the regain of control point.

The display was temporarily occluded two times during the simulation, to mimic the effects of switching attention to secondary tasks, such as checking a cell phone. After the second full display occlusion was cleared, and the participants could see the road again, an acoustic signal—and an intermittent change in background color-notified the drivers to hold the steering wheel (or FCSW) and regain control of the vehicle, ${ }^{5}$ to avoid a collision with a near obstacle, a cuboid of width half of that of the road and 2-m tall.

Although the experiments with the steering wheel and the FCSW were identical, in order to minimize bias effects, the participants were told that both experiments were different. Half of the participants performed the experiment first with the FCSW, while for the other half, the order was reversed (steering wheel first). Both experiments were executed at $50 \mathrm{~km} / \mathrm{h}$. For the case of the FCSW, steering control was implemented with the fractional transfer function (Table IV). In Fig. 5, the various phases of the experiment are shown over the road geometry.

\section{RESULTS}

\section{A. Training Experiments Phase-Day 1}

1) Tracking Experiment \#1: The participants adapted promptly to the test, with the exception of two of them. Participants $P 3$ and $P 4$ found that it was difficult to maintain the degree of torque $( \pm 10.5 \mathrm{~N} \cdot \mathrm{m})$ for $20 \mathrm{~s}$. Nevertheless, once they improved their hand positioning strategy, they also became adapted to the FCSW.

\footnotetext{
${ }^{5}$ The participants had already performed a very similar, but much easier test prior to this one, so they were familiar with all the elements found in the experiment: autonomous driving, temporary occlusion, and regain of control to avoid an object in the center of the road.
} 
2) Tracking Experiment \#2: All the test participants were able to track the target during this exercise. Besides accustoming the participants to FCS, one of the motivating factors for these two initial tests was to determine if humans exhibit arm tremors when holding the FCSW-due to isometric muscle contraction-at particular frequencies. Peaks in the power spectrum at specific frequencies during isometric contraction are reported in the literature, and this has been suggested as evidence of coordinated and rhythmic neural firing [33]. In our data, characteristic peaks at specific frequencies were not found. It is possible that, because the participants used both hands to control the FCSW, hand tremors were filtered out by both hands acting in antiphase and muscle co-contraction. Another possibility is that the rubber element that held the steering wheel fixed partially filtered out the tremors.

\section{B. Performance Measurement Experiments-Day 2}

1) FCS at $30 \mathrm{~km} / \mathrm{h}$ : Fig. 6(a) shows the mean-squared error (MSE) of the lateral offset from the center of the road for each participant and at each phase of the experiment. The figure also includes box plots averaging the results. Because the MSE was fairly similar during the experiment for each transfer function setting (Table V), only the aggregated value for each experimental variant is reported. The responses of $P 4$ were in the outlier range for these experiments, hence not included in the analysis; $P 4$ drove routinely with a large offset from the center of the road with both control devices.

At $30 \mathrm{~km} / \mathrm{h}$, the participants produced larger MSE with the PI transfer function as compared with the baseline transfer function-proportional gain-and the fractional order integrator. Although the second time the PI transfer function appeared, the participants improved their performance, they adapted faster to the fractional order filter. An ANOVA test was performed ( $95 \%$ confidence) to compare the mean performance (MSE) among three groups, corresponding to the three tested transfer functions. The null hypothesis was that the data do not show significant differences among the group means. Although we observed differences among the conditions, these were not significant at $30 \mathrm{~km} / \mathrm{h}:[F(2,42)=$ $0.45, p=0.64]$. This is likely caused by the relatively small sample size, resulting in a low power test.

2) FCS at $50 \mathrm{~km} / \mathrm{h}$ : Fig. 6(b) displays the evolution of the MSE through the different phases of the experiment. The solid dots are the median of the MSE among all the participants in day 1. The box plots summarize the results for the same experiment in day 2; there was a consistent improvement in performance on the second day. The blue line indicates the median performance with a steering wheel. It is noticeable that the participants showed a lower MSE with the FCSW, but this may be related to the characteristics of the employed Logitech steering wheel and the vehicle simulation, which may not necessarily mimic fully realistic driving. However, this was unanticipated, and several participants were surprised by how easy their FCS task was during day 2.

In Fig. 7, the steering movements produced by $P 7$ in this experiment with the FCSW and with the steering wheel (day 1) are compared. For the FCSW, the signal is shown before and after the fractional order filter was applied. The steering

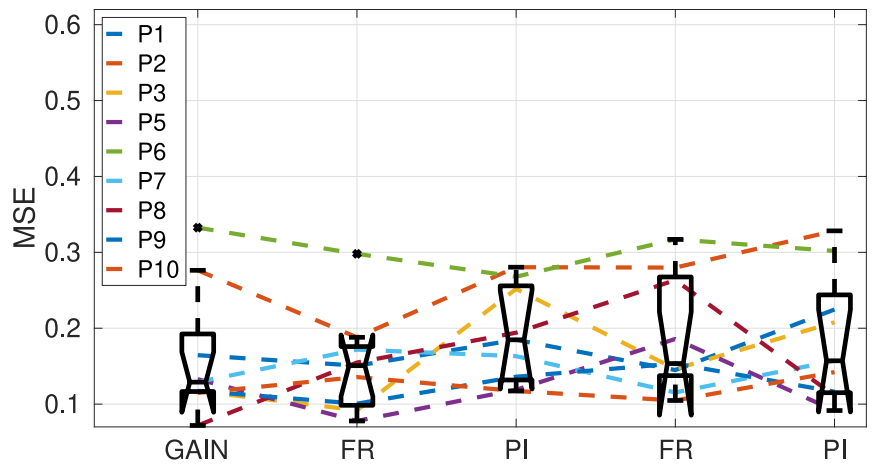

(a)

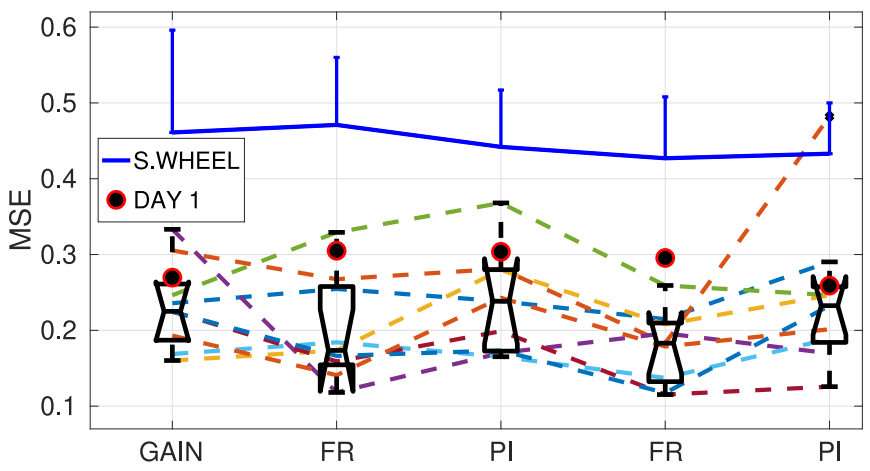

(b)

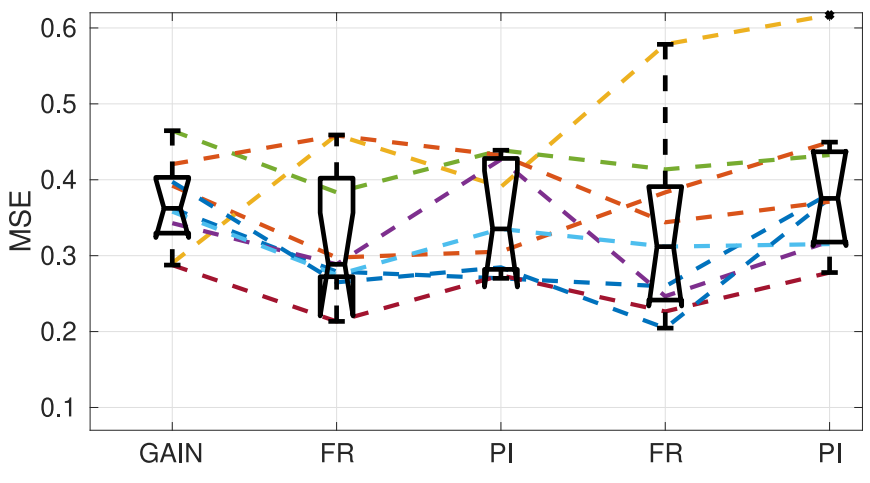

(c)

Fig. 6. MSE for each participant and for each phase of the FCS experiments at (a) $30 \mathrm{~km} / \mathrm{h}$, (b) $50 \mathrm{~km} / \mathrm{h}$, and (c) $70 \mathrm{~km} / \mathrm{h}$. Box plots aggregating the data are also included in each figure. In the box plots, the box edges mark the 25th and 75th percentiles, while the notches indicate $95 \%$ confidence intervals for the median. The box whiskers extend to a maximum of 1.5 the interquartile difference. In (b), median values of the lane-keeping error (MSE) in day 1 and for the same experiment are also shown (solid dots), along with the performance with the Logitech steering wheel (blue line) over the corresponding track segments. The vertical blue lines indicate the variance of the squared errors.

signal in the FCSW is composed of shorter pulses but of higher amplitude than those of the steering wheel. The hypothesis that human operators employ ballistic intermittent corrections while in a control task is a subject of active research [34]. This effect is more clearly manifested when using joysticks or force control devices than when maneuvering with a steering wheel [21].

At $50 \mathrm{~km} / \mathrm{h}$, the differences between the tested transfer functions (Table IV) were larger than those at $30 \mathrm{~km} / \mathrm{h}$; the fractional transfer function yielded the lowest median for the MSE. The PI filter was the worst performer together with the proportional 


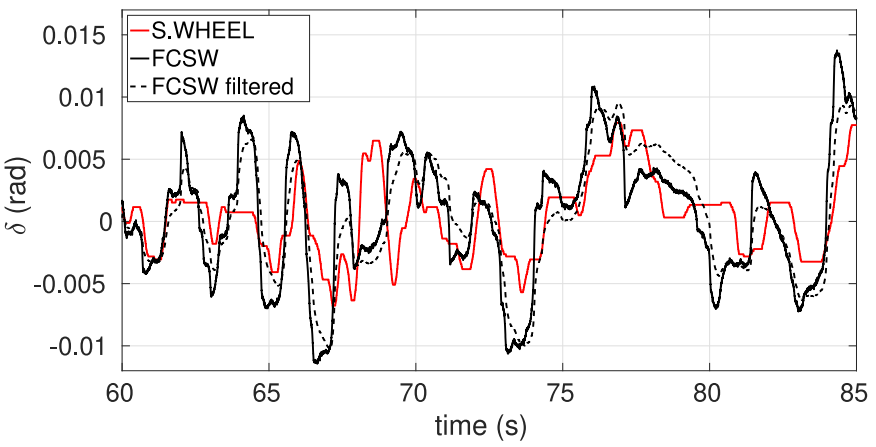

Fig. 7. Comparison of the steering signals from the FCS experiment at $50 \mathrm{~km} / \mathrm{h}$ (day 2), and the driving test with the steering wheel through the same pseudorandomly generated road for $P 7$ and with the fractional order filter (day 1).

transfer function. Adaptation to the PI filter was also worse than at $30 \mathrm{~km} / \mathrm{h}$, although the variance among participants is reduced [Fig. 6(b)]. At $50 \mathrm{~km} / \mathrm{h}$, the ANOVA test yielded a smaller $p$-value than at $30 \mathrm{~km} / \mathrm{h}$, although not yet significant $[F(2,42)=2.55, p=0.09]$.

3) FCS at $70 \mathrm{~km} / \mathrm{h}$ : At this speed, the differences between transfer functions were even more pronounced, but the results were very similar; the proportional and the PI transfer functions were the worse performers [Fig. 6(c)]. The lowest MSE was again obtained with the fractional order filter. In this case, the ANOVA test (95\% confidence) clearly rejected the null hypothesis: $[F(2,42)=5.16, p=0.011]$. Thus, the data suggest statistically significant differences among the tested transfer functions at higher speeds.

4) Regain of Control Experiments: In Fig. 8(a), the path of the vehicle's CG for all the participants and with each control device is displayed-from the time they were requested to regain control by the system. The participants yielded higher distance margins from the obstacle and faster return to the driving lane with the FCSW. All the participants decided to steer tangentially to the road, with the exception of $P 9$ when using the FCSW.

Fig. 8(b) shows the steering movements of $P 6$ with both control devices, and during the obstacle avoidance maneuver; generally, all the participants produced a higher amplitude response in a shorter time with the FCSW. This is most likely caused by the fact the FCSW does not involve arm displacement, nor visual assessment about the current rotation angle of the steering wheel. Furthermore, a $t$-test $(99 \%$ confidence, paired, and one-sided) was performed over the difference in minimum distance to the obstacle among conditions (steering wheel or FCS) $-M=1.81, S D=1.21$. The test shows a statistically significant difference between conditions: $[t(9)=4.74, p=0.00053]$; the recorded data are in agreement with FCS being advantageous under the tested simulated scenario.

\section{Discussion}

\section{A. FCS Experiments Analysis}

Given the observed difference in performance between the fractional and the proportional transfer functions (Fig. 6), it is suggested that filtering becomes more necessary as the

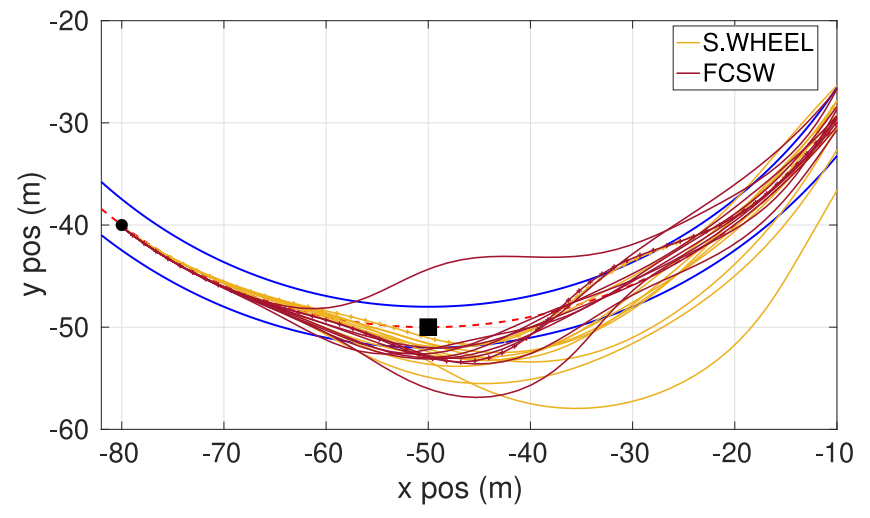

(a)

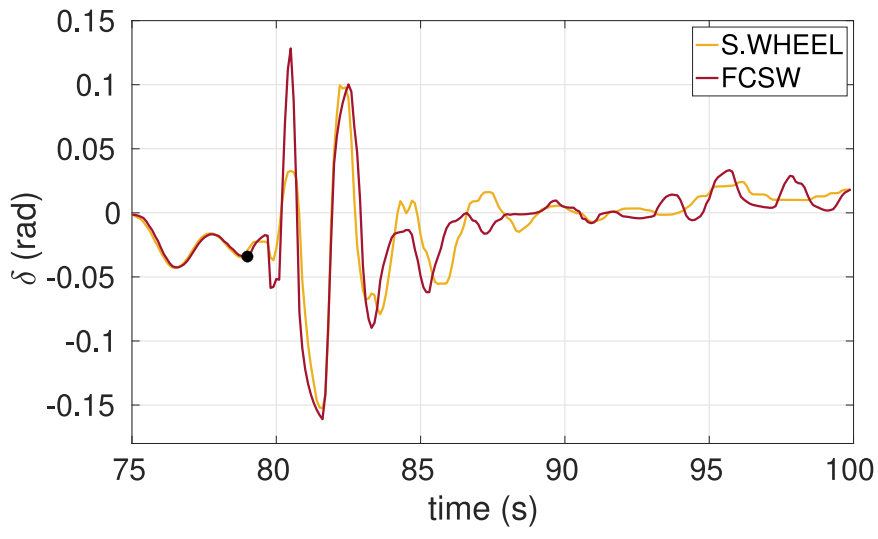

(b)

Fig. 8. (a) Path of the $\mathrm{CG}$ of the vehicle for all the participants in the regain of control experiments (Section II-E4). The blue lines display the position of the left and right boundary lines, while the red dashed line represents the middle lane line. (b) Steering signal in the regain experiments for P6. The black dot marks the point at which the control was transferred back to the driver.

vehicle speed increases. Even so, the extreme memory effect of a classical integral-in the PI transfer function-makes the vehicle more difficult to stabilize at higher speeds, as it is shown in Fig. 6(c) and in the ANOVA test (Section III-B3). Indeed, several participants claimed that the PI controller felt notably lagged. Thus, fractional order filters are reasonable candidates, but other transfer functions not examined here could offer similar performance, such as the second-order or higher order filters and weighted averages. Nevertheless, fractional order filters present the advantage that they model memory effects explicitly, while they still allow for their analysis from a dynamical systems perspective. Furthermore, as a fractional integrator involves only one parameter - aside from the gain constant-it is easily tuned. Another justification is the evidence that the neuromuscular system presents fractionality [27], [30]. As an FCSW involves static control, the viscoelastic response of the muscles may be altered, and a fractional filter could act as a compensator. This is compatible with the fact that at higher speeds, larger fractional integration is needed; humans will hold more tightly a steering wheel when driving under higher workload, increasing their arms mechanical impedance to achieve greater limb stabilization.

With respect to what causes force control to improve performance-for the here reported cases and previous 
literature (Section I-B) - different explanations can be recognized. Response delays are smaller with a joystick than with a steering wheel [30]. A joystick involves a lower ratio between hand movement and control gain than a steering wheel. In the same manner, a force control device reduces the motion required to generate a control pulse to its lowest point. On the other hand, control with a joystick is less precise [35], due to uncomfortable arm positioning-as compared to the steering wheel-and muscle and tendon vibration. The steering signal in the FCSW incorporates some of the features of joystick control; it is composed of shorter pulses but of higher amplitude than those of the steering wheel [Fig. 8(b)]. Thus, the FCSW somewhat combines the comfort of the steering wheel with the rapid control actions of a joystick. This is manifested by the notable results in the regain of control experiment (Section II-E4); the participants avoided the obstacle with larger safety margins and they were able to stabilize the vehicle in a shorter time.

One advantage of FCS is the possibility that the hands may use the control device to stabilize the arms, thus reducing even more hand vibrations and instabilities-at least for the case of laboratory tasks or when controlling vehicles remotely; in [36], it was shown that the viscoelastic properties of muscles are adjusted to produce smooth and stable hand movements through viscoelastic regulation. The same idea was claimed by Kawato in [37]. In [38], it was studied how the CNS increases impedance in unstable directions of arm motion, also with the intent of generating smoother control movements. Thus, a part of any control strategy involves stabilizing the arm itself. With a force controller, arm stabilization is achieved by just holding the device, and all the efforts can be directed toward the control action itself. The reduction of arm vibration has another advantage; it is known that externally induced tendon vibration can alter human's perception on applied force [39], thus a fixed arm position could improve control precision.

Another important advantage is that an operator, controlling a force control device, does not need to look at the hand to assess visually the control input produced by an autonomous system, thus unloading the visual channel of control device supervision. It is known that when the hand is not visible, visual drift occurs [40]. Hence, in low visibility conditions, force control devices may reduce ambiguity in the control responses when regaining control from an autonomous system or interacting with a shared-control system.

\section{B. Critique}

This work does not intend to propose ground vehicles being driven with an FCSW. For example, in semistatic maneuvers such as parking a vehicle, it can be difficult to assess the wheels turning angle based only on steering feel. Indeed, the relation between torque and steering feel is known to be nonlinear [19]. Thus, semistatic manoeuvres have not been tested. Additional challenges are vehicle vibration and the lack of direct feedback on the autonomous system input when in the shared-control mode. Nevertheless, the presented study suggests several implications about force control for highway driving, when using an FCSW as a communication device between the human and an intelligent system assisting in the driving task.

\section{FCS Design Guidelines}

There are a number of schemes, not tested here, in which the FCSW could attain real applicability outside of laboratory tasks. Perhaps the most relevant application is the design of shared-control systems. It has been long recognized that, although vehicle technologies must be aimed at unburdening the human operator, full automation is impractical in many situations [5]. Thus, as humans are better controllers of plants with uncomplicated dynamics, the SBW technology could be employed to reduce driving to a more simple task, in which steering control is shared between the human and an intelligent system.

One of the difficulties in designing shared-control systems is in determining the degree of control that the intelligent system shares with the human. In [41], this concept is referred to as the level of haptic authority (LoHA), and it specifies the resisting force of the control device to the applied force by the human. Thus, the LoHA is set to establish how much of the control task is taken from the human operator by the intelligent system. An analogous and very illustrative concept is that of the $H$-mode [10], which compares shared control to holding the reins of a horse more tightly-manual control from the human operator-or more loosely - highly automated system. This is usually known as the $H$-metaphor.

Normally, the control task at hand and the $H$-mode cannot be considered independent of each other; the impedance of the steering wheel may change the control response of the human, in the same manner as muscle impedance changes the stability of motor control. An FCSW orthogonalizes the control signal from the $H$-mode; thus it is a control device especially well suited to act as a baseline in the implementation of shared-control systems.

An interesting question is what is the role of a steering wheel rotating in a vehicle when in the autonomous driving mode. Thus, a steering wheel could lock itself as an FCSW for highway driving, while the driver can still use it to communicate instructions to the vehicle according to the current $H$-mode. Some of the schemes in which an FCSW may be applicable are summarized as follows.

\section{1) Manual Driving:}

a) Speed Adaptive FCSW: One possibility is a system that smoothly transitions from a steering wheel-at very low speed-to an FCSW for highway driving at higher speeds. Although this has not been tested, the FCSW does not seem a priori appropriate for semistatic manoeuvres, such as driving in a parking lot or in a gas station.

2) Autonomous Driving:

a) Strategic and Maneuvering Levels of Control: According to Michon's model, driving is composed of three levels: i) control; ii) maneuvering; and iii) strategic levels [42]. The FCSW could be used to control only some of these levels. The driver would communicate to the intelligent systemusing the FCSW-the intention of switching lanes 
(maneuvering level) or exiting a highway to reach a destination (strategic level).

b) Low Probability Situation Handling with Full Control: For autonomous driving, it is conceivable an FCSW instead of a moving wheel. In case of a hazard that the automated system cannot handle (such as occluded lane lines), the human driver could bring forth a swifter regain of control with an FCSW. This driving mode would correspond to level 3 automation according to the SAE J3016 automation classification standard.

3) Shared Control:

a) Low Probability Situation Handling With Shared Control: Similar to the case above, the driver could use the FCSW to modify or override the AI control manoeuvres or decisions. Input pulses on the FCSW would be superposed to the control response intended by the intelligent tracking system [43]. For instance, if the intelligent system is handling the road curvature but there is an unexpected hazard on the road (such as a large vehicle partially occupying the adjacent lane), the human driver could add a correction to the undergoing control action. This steering pulse would be relative to the steering angle at the wheels applied by the AI, and hence independent of any visual interpretation of the current steering angle.

b) Shared Control Through H-Mode: With this scheme, the human driver controls the car at all times, but with the help of the intelligent system. The driver can override the control decisions of the AI according to a preset (or adaptive) $H$-mode. FCSWs are more analogous to the reins of a horse than a conventional steering wheel, as the position of the FCSW is independent of the yaw rate. Hence transitions in $H$-mode may be more natural to the driver with an FCSW.

c) Hybrid Mode FCS: Similarly to the manual mode, the steering system could transition between a normal steering wheel and FCS. For example, the system transitions to FCSW when entering an eLane. When the steering system is not locked, this method can be combined with some level of haptic-guidance [7].

An additional consideration is the use of brain-computer interfaces [44] to better implement the intent of the humandriver on the vehicle response.

With respect to the configuration of a steering wheel, one obvious advantage of a circular configuration is its shape invariance to changes in the steering angle; hence it is uncomplicated to perform suitable steering movements even when $\delta>(\pi / 2) \mathrm{rad}$. With an FCSW, this is inconsequential, and one can think about the design of new configurations-for example, the wrist-twist instant steering system included in some Ford prototypes in the past, or the twin-levers in the recent Honda EV-STER model. In this last example, the driver controls the car with two control sticks - one at each hand. The sticks are coupled to each other, hence the antiphase filtering properties of the SW are preserved. This system makes use of the SBW technology to modify the torque in the sticks to neutralize the perturbations produced by lateral acceleration on the driver.

\section{CONCLUSION}

Autonomous driving and shared-control systems are gaining relevance in recent years-partly due to the availability of the $S B W$ technology in conventional cars, AI developments, and an increase in the computational capacity of low-power devices, such as FPGAs. Simultaneously, the evolution of this steering control paradigm opens up new research possibilities toward the design of new steering control devices. In this article, this subject has been analyzed from the prospect of FCS.

Classical literature, in which force control sticks were tested, reports that in some situations, humans perform more efficient control with these devices as compared to conventional control sticks. Herein, these results have been expanded by experiments with human participants, in which they had to control a simulated ground vehicle with an FCSW. The results showed that the human operator adapts promptly to force control and is able to drive a simulated vehicle in a lane-keeping laboratory task.

As the transfer functions-acting as transducers between the applied torque by the human and the steering angle at the wheels of the car-are device and task-dependent, several mappings were evaluated. It was found that fractional transfer functions are suitable and easy to tune candidates and that the order of fractional integration is dependant on the speed of the vehicle. It is hypothesized that this may have some relation to the fact that muscular dynamics are well modeled through fractional differential equations; hence a fractional transfer function yields filtering properties similar to those exhibited by the human neuromuscular system.

In this research, it was also shown that $F C S$ is particularly efficient for the case in which a driver has to regain control back from an autonomous system in a simulated near-collision scenario; the participants were able to avoid an obstacle with larger safety margins and stabilize the vehicle in a shorter time when using the force steering wheel. Although in a real case, the driver may not have enough time to react and avoid a collision, the quicker response may be beneficial in realistic situations, such as taking back control when an autonomous vehicle is drifting off the lane-perhaps due to poorly visible road markings, overriding control from an autonomous parking system or when maneuvering within a gas station.

The generalization of these results from a laboratory task to real-world driving needs further research to assess potential shortcomings, such as vehicle induced hand vibration and lack of interpretability on applied torque when at very slow speeds or semistatic maneuvering. Nevertheless, guidelines toward attainable implementations concerning FCS in highway driving are enclosed in this article. An example is using the force control device to handle the vehicle only at the maneuvering and strategic levels, while a driver model handles lane keeping at the control level.

As this article was the first study on FCS, the number of participants was relatively small. An analytical examination 
discussed designed guidelines_-including alternative steering wheel designs, such as twin-levers, additional transfer functions (perhaps self-tunable [45]), speed control, and a larger sample size_-is left for future research.

\section{REFERENCES}

[1] D. A. Abbink et al., "A topology of shared control systems-Finding common ground in diversity," IEEE Trans. Human-Mach. Syst., vol. 48, no. 5, pp. 509-525, Oct. 2018.

[2] C. Zhang, S. Bengio, M. Hardt, B. Recht, and O. Vinyals, "Understanding deep learning requires rethinking generalization," 2016. [Online]. Available: arXiv:1611.03530.

[3] D. Song et al., "Physical adversarial examples for object detectors," in Proc. 12th USENIX Workshop Offensive Technol. (WOOT), 2018, p. 1.

[4] A. Toffetti et al., "CityMobil: Human factor issues regarding highly automated vehicles on elane," Transp. Res. Rec. J. Transp. Res. Board, vol. 2110, no. 1, pp. 1-8, 2009. [Online]. Available: https://journals.sagepub.com/doi/abs/10.3141/2110-01

[5] H. Birmingham and F. Taylor, "A design philosophy for man-machine control systems," Proc. IRE, vol. 42, no. 12, pp. 1748-1758, Dec. 1954

[6] J. C. Licklider, "Man-computer symbiosis," IRE Trans. Hum. Factors Electron., vol. HFE-1, no. 1, pp. 4-11, Mar. 1960.

[7] A. Ranasinghe, N. Sornkarn, P. Dasgupta, K. Althoefer, J. Penders, and T. Nanayakkara, "Salient feature of haptic-based guidance of people in low visibility environments using hard reins," IEEE Trans. Cybern. vol. 46, no. 2, pp. 568-579, Feb. 2016.

[8] W. Mugge, I. A. Kuling, E. Brenner, and J. B. Smeets, "Haptic guidance needs to be intuitive not just informative to improve human motor accuracy," PLOS ONE, vol. 11, no. 3, 2016, Art. no. e0150912.

[9] F. Flemisch, C. Adams, S. Conway, K. Goodrich, M. Palmer, and M. Schutte, "The h-metaphor as a guideline for vehicle automation and interaction," NASA Langley, Langley, VA, USA, Rep. NASA/TM-2003212672, 2003

[10] F. Flemisch et al., "Shared and cooperative control of ground and air vehicles: Introduction and general overview," in Proc. IEEE Int. Conf. Syst. Man Cybern. (SMC), Banff, AB, Canada, 2017, pp. 858-863.

[11] J. A. Michon, "A critical view of driver behavior models: What do we know, what should we do?" in Human Behavior and Traffic Safety. Boston, MA, USA: Springer, 1985, pp. 485-524. [Online]. Available: https://link.springer.com/chapter/10.1007\%2F978-1-4613-2173-6_19

[12] C. Gibbs, "The continuous regulation of skilled response by kinaesthetic feed back," Brit. J. Psychol. Gen. Section, vol. 45, no. 1, pp. 24-39, 1954.

[13] D. T. McRuer and R. Magdaleno, "Human pilot dynamics with various manipulators," Syst. Technol. Inc, Hawthorne CA, USA, Rep. AFFDL TR 66-138, 1966.

[14] S. K. Card, W. K. English, and B. J. Burr, "Evaluation of mouse, ratecontrolled isometric joystick, step keys, and text keys for text selection on a crt," Ergonomics, vol. 21, no. 8, pp. 601-613, 1978.

[15] J. D. Rutledge and T. Selker, "Force-to-motion functions for pointing," in Proc. IFIP TC13 3rd Int. Conf. Hum. Comput. Interaction, 1990, pp. 701-706.

[16] R. C. Barrett, E. Selker, J. Rutledge, and R. Olyha, "Negative inertia: A dynamic pointing function," in Proc. Conf. Companion Hum. Factors Comput. Syst., 1995, pp. 316-317.

[17] A. Tustin, "The nature of the operator's response in manual control, and its implications for controller design," J. Inst. Elect. Eng. IIA, Autom. Reg. Servo Mech., vol. 94, no. 2, pp. 190-206, May 1947.

[18] J. W. Hegg, M. P. Smith, and L. Yount, "Sidestick controllers for advanced aircraft cockpits," in Proc. IEEE/AIAA 11th Digit. Avionics Syst. Conf., Seattle, WA, USA, 1992, pp. 491-499.

[19] A. Newberry, M. Griffin, and M. Dowson, "Driver perception of steering feel," Proc. Inst. Mech. Eng. D, J. Automobile Eng., vol. 221, no. 4, pp. $405-415,2007$.

[20] N. Merat and A. H. Jamson, "How do drivers behave in a highly automated car," in Proc. 5th Int. Driving Symp. Hum. Factors Driver Assess. Training Veh. Design, 2009, pp. 514-521.

[21] M. Martínez-García, Y. Zhang, and T. Gordon, "Modeling lane keeping by a hybrid open-closed-loop pulse control scheme," IEEE Trans. Ind. Informat., vol. 12, no. 6, pp. 2256-2265, Dec. 2016.
[22] D. Shreiner and B. T. K. O. A. W. Group, OpenGL Programming Guide: The Official Guide to Learning OpenGL, Versions 3.0 and 3.1. Ann Arbor, MI, USA: Pearson Educ., 2009.

[23] J. Y. Chen and J. E. Thropp, "Review of low frame rate effects on human performance," IEEE Trans. Syst., Man, Cybern. A, Syst. Humans, vol. 37, no. 6, pp. 1063-1076, Nov. 2007.

[24] J. D. Lambert, Numerical Methods for Ordinary Differential Systems: The Initial Value Problem. Chichester, U.K.: Wiley, 1991.

[25] M. Abe, Vehicle Handling Dynamics: Theory and Application. Amsterdam, The Netherlands: Butterworth-Heinemann, 2015.

[26] I. Podlubny, Fractional Differential Equations: An Introduction to Fractional Derivatives, Fractional Differential Equations, to Methods of Their Solution and Some of Their Applications, vol. 198. San Diego, CA, USA: Academic, 1998.

[27] R. L. Magin, Fractional Calculus in Bioengineering. Redding, CA, USA: Begell House, 2006.

[28] M. Martínez-García, Y. Zhang, and T. Gordon, "Memory pattern identification for feedback tracking control in human-machine systems," Hum. Factors, to be published. [Online]. Available: https://doi.org/10.1177/0018720819881008

[29] R. Magin, B. Vinagre, and I. Podlubny, "Can cybernetics and fractional calculus be partners?: Searching for new ways to solve complex problems," IEEE Syst., Man, Cybern. Mag., vol. 4, no. 3, pp. 23-28, Jul. 2018

[30] M. Martínez-García, T. Gordon, and L. Shu, "Extended crossover model for human-control of fractional order plants," IEEE Access, vol. 5, pp. 27622-27635, 2017.

[31] K. Perlin, "An image synthesizer," ACM SIGGRAPH Comput. Graph., vol. 19 , no. 3, pp. 287-296, 1985.

[32] D. D. Salvucci and R. Gray, "A two-point visual control model of steering," Percept. London, vol. 33, no. 10, pp. 1233-1248, 2004.

[33] J. McAuley, J. Rothwell, and C. Marsden, "Frequency peaks of tremor, muscle vibration and electromyographic activity at $10 \mathrm{~Hz}, 20 \mathrm{~Hz}$ and $40 \mathrm{~Hz}$ during human finger muscle contraction May reflect rhythmicities of central neural firing," Exp. Brain Res., vol. 114, no. 3, pp. 525-541, 1997.

[34] A. Zgonnikov and G. Markkula, "Evidence accumulation account of human operators' decisions in intermittent control during inverted pendulum balancing," in Proc. IEEE Int. Conf. Syst. Man Cybern. (SMC), Miyazaki, Japan, 2018, pp. 716-721.

[35] B. Andonian, W. Rauch, and V. Bhise, "Driver steering performance using joystick vs. steering wheel controls," SAE Trans., vol. 112, no. 6, pp. 1-12, 2003.

[36] H. Gomi and R. Osu, "Task-dependent viscoelasticity of human multijoint arm and its spatial characteristics for interaction with environments," J. Neurosci., vol. 18, no. 21, pp. 8965-8978, 1998.

[37] M. Kawato, "Internal models for motor control and trajectory planning," Current Opinion Neurobiol., vol. 9, no. 6, pp. 718-727, 1999.

[38] E. Burdet, R. Osu, D. W. Franklin, T. E. Milner, and M. Kawato, "The central nervous system stabilizes unstable dynamics by learning optimal impedance," Nature, vol. 414, no. 6862, pp. 446-449, 2001.

[39] L. Jones and I. Hunter, "Effect of muscle tendon vibration on the perception of force," Exp. Neurol., vol. 87, no. 1, pp. 35-45, 1985.

[40] M. Botvinick and J. Cohen, "Rubber hands 'feel' touch that eyes see," Nature, vol. 391, no. 6669, p. 756, 1998.

[41] D. A. Abbink, M. Mulder, and E. R. Boer, "Haptic shared control: Smoothly shifting control authority?" Cogn. Technol. Work, vol. 14, no. 1, pp. 19-28, 2012.

[42] T. Gordon and K. Srinivasan, "Modeling human lane keeping control in highway driving with validation by naturalistic data," in Proc. IEEE Int. Conf. Syst. Man Cybern. (SMC), San Diego, CA, USA, 2014, pp. 2507-2512.

[43] D. Wang, L. Cheng, and J. Yan, "Self-learning robust control synthesis and trajectory tracking of uncertain dynamics," IEEE Trans. Cybern., early access, Mar. 27, 2020, doi: 10.1109/TCYB.2020.2979694.

[44] B. Wang et al., "Common spatial pattern reformulated for regularizations in brain-computer interfaces," IEEE Trans. Cybern., early access, Apr. 22, 2020, doi: 10.1109/TCYB.2020.2982901.

[45] X. Gu, Q. Shen, and P. P. Angelov, "Particle swarm optimized autonomous learning fuzzy system," IEEE Trans. Cybern., early access, Feb. 20, 2020, doi: 10.1109/TCYB.2020.2967462. 


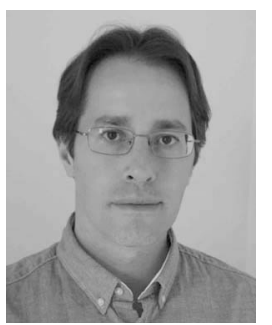

Miguel Martínez-García received the B.Sc. degree in mathematics and the M.Sc. degree in advanced mathematics and mathematical engineering from the Polytechnic University of Catalonia, Barcelona, Spain, in 2013 and 2018, respectively.

$\mathrm{He}$ is a Lecturer of human-machine systems with Loughborough University, Loughborough, U.K. He has been also worked as a Researcher with the University of Lincoln, Lincoln, U.K., since 2017 and the Advanced Virtual Reality Research Centre, Loughborough University. His research interests include human-machine integration, machine learning, artificial intelligence, intelligent signal processing, and complex systems, with particular focus in the analysis of nonlinear signals representing phenomena of interest between humans and machines.

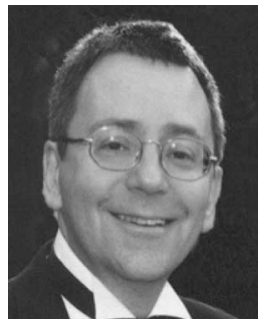

Roy S. Kalawsky received the B.Sc., M.Sc., and Ph.D. degrees from the University of Hull, Hull, U.K., in 1978, 1984, and 1991, respectively.

He is the Director of the Advanced VR Research Centre, Loughborough University, Loughborough, U.K. He has extensive industrial and academic experience in systems engineering spanning over 32 years. He spent over 17 years working for BAE Systems and was responsible for Advanced Crew Station research across the Military Aircraft Division. In 1995, he joined Loughborough University and established the Advanced VR Research Centre.

Dr. Kalawsky is a Fellow of IET and the Royal Society of Arts. He is also a Chartered Engineer.

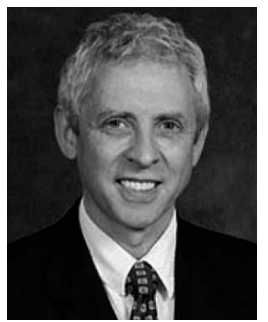

Timothy Gordon (Member, IEEE) received the B.A. and M.A. degrees in mathematics from the University of Cambridge, Cambridge, U.K., in 1974 and 1975, respectively, and the Ph.D. degree in applied mathematics from the Department of Applied Mathematics and Theoretical Physics, University of Cambridge, in 1983.

$\mathrm{He}$ is currently a Professor with the School of Engineering, University of Lincoln, Lincoln, U.K., and has held several senior academic posts, including a Professor of mechanical engineering with the University of Michigan at Ann Arbor, Ann Arbor, MI, USA, and the Ford Professor of automotive engineering with Loughborough University, Loughborough, U.K.

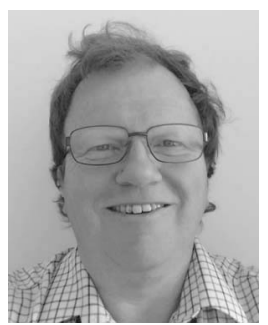

Tim Smith received the B.Eng. degree in electrical and electronic engineering from Sheffield University, Sheffield, U.K., in 1982, and the M.Sc. degree in computer science from Bristol Polytechnic, Bristol, U.K., in 1989.

He joined BAE Dynamics, London, U.K., where he was engaged in the research and development of future radar and thermoelectric tracking and guidance systems. In 2012, he joined the University of Lincoln, Lincoln, U.K., on the Multibody Advanced Airship for Transport Program. His current research interests include hybrid electrical energy storage, electric air vehicles, and integrated electrical-aerostructures.

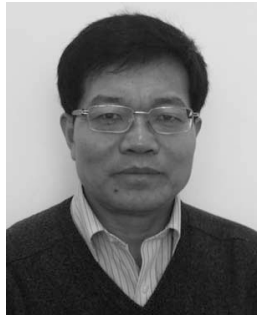

Qinggang Meng (Senior Member, IEEE) received the B.S. and M.S. degrees in electronic engineering from Tianjin University, Tianjin, China, in 1989 and 1994, respectively, and the Ph.D. degree in computer science from the University of Wales, Aberystwyth, U.K., in 2003.

$\mathrm{He}$ is currently a Professor of robotics and AI with the Department of Computer Science, Loughborough University, Loughborough, U.K. His research interests include biologically inspired learning algorithms and developmental robotics, service robotics, agricultural robotics, robot learning and adaptation, multi-UAV cooperation, human motion analysis and activity recognition, activity pattern detection, pattern recognition, artificial intelligence, computer vision, and embedded intelligence.

Prof. Meng is a Fellow of the Higher Education Academy, U.K.

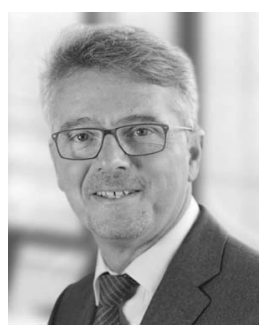

Frank Flemisch received the Dipl.-Ing. degree in aerospace engineering with a specialization in system dynamics and the Dr.-Ing. degree in human factors from the University of Armed Forces, Munich, Germany, in 1989 and 2000, respectively.

$\mathrm{He}$ started as an Aerospace Engineer with a specialization in systems engineering and system dynamics. He spent many years in research on assistant systems and automation with the University of Munich, Munich; NASA, Washington, DC, USA; and DLR, Cologne, Germany, and served as the lead of a national standardization group and a technical expert in ISO TC204. Since 2011, he has been leading a Department of Human System Integration, Fraunhofer Institute for Communication, Information Processing and Ergonomics FKIE, Wachtberg, Germany. He is a Professor of human systems integration with RWTH Aachen University, Aachen, Germany, and a member of the NATO-STO Human Factors and Medicine Panel. 